\title{
Vases clos et systèmes : Les enjeux de la régionalisation des soins de santé
}

\author{
par Scot H Simpson
}

L a régionalisation fait référence à la création d'une structure d'administration et de gouvernance intermédiaire (souvent appelée régie régionale ou conseil régional de la santé) qui est responsable de l'organisation et de la prestation des services de soins de santé au sein d'une population définie ${ }^{1}$. La formation d'un conseil régional de la santé écarte des organismes individuels (comme les conseils d'administration des hôpitaux communautaires) et du ministère de la Santé provincial la responsabilité des décisions de financement et d'affectation des ressources de soins de santé. Les principaux objectifs de la régionalisation sont de contribuer à contrôler les coûts croissants des soins de santé (au moyen d'économies d'échelle), d'accroître la capacité de réaction et la responsabilité envers la population en ce qui a trait aux besoins en matière de santé et de rehausser la participation $\mathrm{du}$ public dans les décisions en santé ${ }^{1}$. Essentiellement, la responsabilité de la prestation des services de soins de santé passe des organisations indépendantes, fragmentées en vases clos (un exemple : avant la régionalisation en Saskatchewan, la province comptait plus de 400 organismes sanitaires indépendants, comme des hôpitaux de soins de courte durée et des groupes de soins communautaires ${ }^{2}$ ) vers un "système " de soins de santé assurés de façon intégrée par un seul organisme régional.

La régionalisation de la prestation des soins de santé a commencé à la fin des années 1980, début des années 1990 dans l'ensemble des provinces, à l'exception de l'Ontario ${ }^{3}$. Des projets de régionalisation étaient mis de l'avant en réponse à des observations issues d'un nombre de commissions, de groupes de travail et de tribunes publiques à l'échelle fédérale et provinciale visant à trouver des moyens d'améliorer le système de santé1. Chaque province a créé des conseils régionaux de la santé, dont le nombre, l'étendue des responsabilités et les membres varient. Alors que certaines provinces ont des conseils distincts pour superviser les services aux établissements et à la communauté, la plupart ont des conseils régionaux de la santé qui ont tout un éventail de responsabilités, notamment : cliniques médicales, prévention et promotion de la santé, services aux jeunes et aux familles, santé publique, soins en cancérologie, santé mentale, hôpitaux, et centres d'hébergement et de soins de longue durée 3 .
Dans la plupart des provinces, les membres des conseils régionaux de la santé sont nommés par le ministre provincial de la Santé.

Bien qu'on reconnaisse l'existence d'un certain nombre de difficultés associées au fonctionnement efficace des conseils régionaux de la santé, je ne m’attarderai qu’à quatre de celles-ci. L'une des difficultés fondamentales est la nécessité de caractériser l'importance relative d'une large gamme de besoins en matière de soins de santé au sein d'une région. Les partisans de la régionalisation croyaient que celle-ci créerait une équité dans l'allocation des services de santé; or, avec la fermeture de nombreux hôpitaux de petites localités et un recentrage des pouvoirs vers les centres urbains, certains remettent en question cette idée ${ }^{4}$. Pour contribuer à la prise de décisions appropriées et équitables, les conseils régionaux de la santé devraient idéalement recourir à des formules de financement valides et dynamiques 5 . Toutefois, de telles formules requièrent de grandes quantités de données individuelles, ce qui met en perspective la seconde difficulté : bien que les données individuelles nous permettent de définir et de tenir compte d'importants facteurs qui influencent les besoins en soins de santé, il est souvent difficile d'obtenir des données épidémiologiques fiables et de grande qualitét. Ironiquement, les ressources humaines et l'infrastructures nécessaires pour collecter et interpréter efficacement cette information pourraient en fait exacerber le problème des coûts élevés des soins de santé1.

Une troisième difficulté au fonctionnement efficace des conseils régionaux de la santé est le fait que beaucoup d'entre eux n'ont que peu de contrôle sur les principales dépenses, comme les honoraires des médecins et le coût des médicaments, et un pouvoir limité sur la création de politiques ${ }^{4}$. Faute de délégation de pouvoir de la part des ministres provinciaux de la Santé, les conseils régionaux deviennent une autre couche de l'administration du système de soins de santé. Finalement, la représentation du public aux conseils régionaux de la santé est peut-être l'un des plus grands défis jamais rencontrés. Bien que l'intention d'avoir une telle représentation soit louable, le choix des membres du public peut s'avérer difficile. Par exemple, si 
seulement un membre du public participe à un conseil particulier, il est impératif de démontrer que la personne choisie exprime les besoins de l'ensemble des résidents de la région. La participation d'un membre du public ayant un intérêt particulier pour un nombre limité de sujets peut être préjudiciable non seulement au processus de prise de décision interne du conseil, mais aussi à la perception hors des rangs du conseil quant à son mode de prise de décision ${ }^{7}$.

Depuis leur arrivée, il y a vingt ans, les conseils régionaux de la santé ont connu de nombreux ajustements. Certains, en réponse aux pressions publiques ou politiques ou dans une tentative de résoudre quelques-unes des difficultés mentionnées plus haut. Dans certaines provinces, le nombre de conseils a varié, tout comme leurs limites géographiques, l'étendue de leurs responsabilités et la composition de ses membres. Il est clair qu’il n'y a pas de solution facile à l'organisation et à la prestation adéquates des services de soins de santé. Une chose est incontestable cependant : la régionalisation a un impact sur la façon dont nous prodiguons les soins à nos patients.

Dans ce numéro du $J C P H$, les auteurs de la chronique Le pour et le contre se penchent justement sur les bons et moins bons aspects de différentes approches à la régionalisation et des conséquences de ces dernières sur le fonctionnement des services de pharmacie dans les établissements de santé. ${ }^{8,9}$ Un seul service de pharmacie régionalisé aide-t-il ou nuit-il à l'avancement de notre profession? De façon plus globale, la régionalisation du système de soins de santé a-t-elle tenu ses promesses de contrôle des coûts, de meilleure capacité de réaction et d'obligation de rendre des comptes pour ce qui est des besoins de la population, et d'accroissement de la participation du public dans votre province? Je vous laisse, chers lecteurs, le soin de répondre.

[Traduction par l'éditeur]

\section{References}

1. Church J, Barker P. Regionalization of health services in Canada: a critical perspective. Int J Health Serv 1998;28(3):467-486.

2. Marchildon GP. Regionalization and health services restructuring in Saskatchewan [article présenté à une conférence]. Dans : Health services restructuring: new evidence and new directions; 17 novembre 2005; Kingston (ON). Montréal (QC): Institut de recherche en politiques publiques; 2005. Publié au www.irpp.org/events/archive/nov05JDI/ marchildon.pdf

3. Les soins de santé au Canada. Ottawa (ON). Institut canadien d'information sur la santé; 2005.

4. Collier R. Is regionalization working? CMAJ 2010;182(4):331-332.

5. Smith PC, Rice N, Carr-Hill R. Capitation funding in the public sector. $J R$ Stat Soc Ser A Stat Soc 2001;164(2):217-257.

6. Hurley J. Regionalization and the allocation of healthcare resources to meet population health needs. Healthc Pap 2004;5(1):34-39.

7. Abelson J, Forest PG, Eyles J, Smith P, Martin E, Gauvin FP. Obtaining public input for health-systems decision-making: past experiences and future prospects. Can Public Adm 2002;45(1):70-97.

8. Slobodan J, van Haaften D, Chambers CR. Is a single provincial pharmacy program beneficial for the advancement of pharmacy practice? The "pro" side. Can J Hosp Pharm 2011;64(4):285-286.

9. Wilson B. Is a single provincial pharmacy program beneficial for the advancement of pharmacy practice? The "con" side. Can J Hosp Pharm 2011;64(4):286-287.

Scot H Simpson, B.S.P., Pharm. D., M. Sc., est professeur agrégé de la Faculté de pharmacie et des sciences pharmaceutiques de l'Université de l'Alberta, à Edmonton, en Alberta. II est également rédacteur adjoint du JCPH.

Adresse de correspondance :

D Scot H Simpson

Faculty of Pharmacy and Pharmaceutical Sciences

2125A Dentistry/Pharmacy Centre

University of Alberta

Edmonton (AB) T6G 2N8

Courriel : ssimpson@pharmacy.ualberta.ca 